\title{
A novel hypothermic machine perfusion system using a LifePort Kidney Transporter for the preservation of rat liver
}

\author{
CHENG ZENG ${ }^{1-3}$, XIAOYAN HU ${ }^{1-3}$, YANFENG WANG ${ }^{1-3}$, \\ XIANPENG ZENG ${ }^{1-3}$, YAN XIONG ${ }^{1-3}$, LING LI $^{1-3}$ and QIFA YE ${ }^{1-4}$ \\ ${ }^{1}$ Transplant Center, Zhongnan Hospital, Wuhan University; ${ }^{2}$ Institute of Hepatobiliary Diseases, Wuhan University; \\ ${ }^{3}$ Hubei Key Laboratory of Medical Technology on Transplantation, Zhongnan Hospital, Wuhan University, Wuhan, \\ Hubei 430071; ${ }^{4}$ Research Center of National Health Ministry on Transplantation Medicine Engineering and \\ Technology, The Third Xiangya Hospital of Central South University, Changsha, Hunan 410013, P.R. China
}

Received December 22, 2016; Accepted July 27, 2017

DOI: 10.3892/etm.2017.5587

\begin{abstract}
The protective mechanisms for liver preservation associated with hypothermic machine perfusion (HMP) remain unclear. However, the lack of a common and portable HMP system for rat livers limits the study of HMP. The present study aimed to develop a novel, modified HMP system using a LifePort Kidney Transporter for preserving rat livers. A simple ' $\mathrm{Y}$ ' shunt combined with a pressoreceptor for flow and pressure regulation was adapted to perfuse rat livers via the portal vein continuously using a LifePort Kidney Transporter under its 'prime mode' setting. An electronic scale was installed under the liver container to calculate the portal inflow according to the association with weight, density and volume of the perfusate. A total of 10 rat livers underwent $6 \mathrm{~h}$ of HMP using histidine-tryptophan-ketoglutarate solution enriched with acridine orange (AO) and propidium iodide (PI). The perfusion status of HMP was assessed by comparison of AO+PI-positive cell count in core region (CR) and peripheral region (PR) of rat liver under fluorescence microscopy. The dynamics (inflow, pressure and intrahepatic resistance of perfusion) were assessed to identify whether this system met the demands for HMP of rat livers. Biochemical [alanine transaminase (ALT), lactate dehydrogenase (LDH) and endothelin levels] and histological parameters (sinusoidal dilatation, endothelial cell detachment and vacuolization) were measured to determine cellular damage associated with HMP. No significant difference was observed between the CR and PR according to the comparison of the AO+PI-positive cell count, which indicated
\end{abstract}

Correspondence to: Dr Qifa Ye, Transplant Center, Zhongnan Hospital, Wuhan University No. 169 Donghu Road, Wuhan, Hubei 430071, P.R.China

E-mail: yqf_china@163.com

Key words: hypothermic machine perfusion, preservation, liver transplantation that complete perfusion was achieved. Intrahepatic resistance significantly decreased during the initial $3 \mathrm{~h}$ of $\operatorname{HMP}(\mathrm{P}<0.01)$, but remained stable during the final $3 \mathrm{~h}$. ALT and LDH levels significantly increased over the $6 \mathrm{~h}$ HMP duration: ALT $(0 \mathrm{~h}$, $42.67 \pm 5.81 \mathrm{U} / 1 ; 3 \mathrm{~h}, 90.67 \pm 6.74 \mathrm{U} / 1 ; 6 \mathrm{~h}, 164.33 \pm 7.31 \mathrm{U} / \mathrm{l}$; $\mathrm{P}<0.01)$ and $\mathrm{LDH}(0 \mathrm{~h}, 492.90 \pm 90.20 \mathrm{U} / 1 ; 3 \mathrm{~h}, 973.53 \pm 97.4$; $6 \mathrm{~h}, 1,843.40 \pm 85.78 \mathrm{U} / \mathrm{l} ; \mathrm{P}<0.01)$ However, the levels of endothelin and oxygen consumption were constant throughout HMP. Furthermore, histological analysis indicated sinusoidal dilation was significantly increased in the post-HMP group compared with the pre-HMP group $(\mathrm{P}<0.01)$; however, no other significant differences were observed. Combined with the results of ATP test $(640.64 \pm 29.46 \mathrm{nmol} / \mathrm{l})$ and bile production $(4.88 \pm 0.69 \mu \mathrm{l} / \mathrm{h} / \mathrm{g}$ of liver) at the end of HMP, the present results demonstrated minimal cellular injury associated with HMP while retaining the dependability and portability of the LifePort Kidney Transporter, which suggests the modified HMP system met the demands required and may be suitable for rat liver preservation.

\section{Introduction}

To overcome the shortage of brain-dead donors for liver transplantation, extended criteria donors (ECDs) have been used (1). ECDs have been considered as potential donors for $>20$ years (2). ECDs include livers from older donors $(>60$ years) $(3,4)$, donors of cardiac death $(5)$ or those with steatosis livers exceeding traditional limits $(>30 \%)(6)$. These grafts are known to be more sensitive to ischemia/reperfusion injury (IRI) following transplantation due to their pathological changes (including impairment of liver function associated with age changes, warm ischemia injury prior to procurement and moderate or severe hepatocyte steatosis) (3-6).

Nowadays, it has been demonstrated that cold storage, a traditional organ preservation technique, cannot meet the demands of ECD preservation due to the reduced ischemic tolerance of these marginal grafts $(7,8)$. Compared with cold storage, hypothermic machine perfusion (HMP), a dynamic preservation technique, results in continuous delivery of oxygen for cellular energy reconstitution, supply of metabolic substrates, washout of residual erythrocytes, thrombi and toxic 
metabolic byproducts. These advantages of HMP are helpful to improve the quality of ECDs. Moreover, the graft viability can be assessed by the perfusate tests and perfusion indexes (9). Although a number of previous clinical and experimental studies have reported that HMP may attenuate IRI in organs more effectively than cold storage $(1,10-12)$, the underlying mechanisms have not been clearly identified. Unlike large animals, including pigs and rabbits, rats are best-suited for studying mechanisms associated with HMP due to the fact that a large data base from studies of rat livers enables effective comparison of observations; furthermore, the relatively low cost and low maintenance is beneficial (13). However, the major obstacle preventing large-scale experimental application of HMP for rat livers is a lack of a portable HMP system that is compatible with the size of rat livers. The LifePort Kidney Transporter is a type of portable and automatic HMP system for kidney preservation $(14,15)$. However, the pulsatile perfusion and size of the piping system is not suitable for rat livers. In the present study the development of a novel HMP system using a LifePort Kidney Transporter for rat livers was conducted to demonstrate its feasibility for rat liver preservation.

\section{Materials and methods}

HMP system based on LifePort for rat livers. According to the preliminary test from LifePort, the 'Prime' mode was used to perform continuous perfusion via the portal vein, indicated by the oscillogram with a high frequency and small wave range (Fig. 1A). An image of the modified LifePort system for HMP of rat livers is indicated in Fig. 1B and C. The system consisted of a rat liver container for perfusion that was installed in the sterile drape of the LifePort Kidney Transporter 1.0 (Organ Recovery system, Inc., Chicago, IL, USA). A self-made ' $Y$ ' shunt was constructed to communicate between the infuse line and the pressoreceptor of BL-420F Biological Functional System (Chengdu Taimeng Science and Technology Co., Ltd., Chengdu, China) that was connected to the rat portal vein cannula (inner diameter, $1.8 \mathrm{~mm}$; outer diameter, $2.1 \mathrm{~mm}$ ) to measure perfusion pressure. The self-made ' $\mathrm{Y}$ ' shunt was constructed of polyethylene and had three sides named as A, B and C. C-side was connected to the infuse line and $\mathrm{A}$-side was connected to the pressoreceptor. B-side was the shunt side for decreasing the portal inflow and avoiding termination of LifePort due to excessive pressure under Prime mode. A and B-sides each contained a valve to adjust the flow, which are indicated as ' 1 ' and ' 2 ', respectively. An electronic scale was installed under the liver container to record the increase of weight per min. The density of histidine-tryptophan-ketoglutarate (HTK; Dr Franz Koehler Chemie GmbH, Bensheim, Germany) solution at $4^{\circ} \mathrm{C}$ was $1.06 \pm 0.01 \mathrm{~g} / \mathrm{ml}$. Subsequently, the portal inflow per min $(\mathrm{ml} / \mathrm{min})$ was gravimetrically estimated.

Animals. A total of 10 adult male Sprague Dawley rats (age, 8-10 weeks; weight, 250-300 g) were purchased from Experimental Animal Culture Center of Hubei Centers for Disease Control (Wuhan, China). All of the animals were housed under standard laboratory conditions (temperature $25 \pm 2^{\circ} \mathrm{C}$, relative humidity $55 \pm 5 \%$ and a 12 -h light/dark cycle) and had free access to food and water. All the animal experiments were approved by the Animal Ethics Committee of Wuhan University (Wuhan, China) and rats were treated in accordance with the Experimental Animal Regulations of the People's Republic of China and the Guide for the Care and Use of Laboratory Animals of the USA.

Experimental procedure. Rats were anesthetized with intraperitoneal injection of $10 \%$ chloral hydrate $(300 \mathrm{mg} / \mathrm{kg}$; Sinopharm Group Co., Ltd., Shanghai, China). Livers were harvested from rats prior to cardiac death to avoid warm ischemia injury to livers, as described previously (16). Once the abdomen was exposed by performing a midline incision and the liver was freed from the ligamentous attachments, the common bile duct was cannulated using an epidural guiding tube (Jiangsu Changfeng Medical Industry Co., Ltd., Jiangsu, China) for collection of the total bile production during HMP.

An intravenous injection of $2 \mathrm{ml}$ saline with $100 \mathrm{U}$ heparin (Nanjing Jiancheng Bioengineering Institute, Nanjing, China) via the right iliac vein was performed for heparinization (16). Subsequently, the hepatic artery was ligated and the portal vein was cannulated using a poly ethylene catheter (outer diameter, $2.1 \mathrm{~mm}$; inner diameter, $1.8 \mathrm{~mm}$ ). The livers were flushed in situ with $20 \mathrm{ml} \mathrm{HTK}$ solution at $4^{\circ} \mathrm{C}$ (5 min) and harvested. Another poly ethylene catheter (outer diameter, $3.5 \mathrm{~mm}$; internal diameter, $3 \mathrm{~mm}$ ) was inserted into the suprahepatic inferior vena cava to collect the hepatic effluent (17). Subsequently, rats were sacrificed via intraperitoneal administration of $130 \mathrm{mg} / \mathrm{kg}$ sodium pentobarbital (Shanghai Pharmaceuticals Holding Co., Ltd., Shanghai, China).

Following the manufacturer's protocol for the LifePort Kidney Transporter, the temperature of the circuit was decreased to $0-4^{\circ} \mathrm{C}$. Once the circuit was primed, the liver was connected and ready for perfusion under prime mode. HMP was performed for $6 \mathrm{~h}$ via the portal vein using HTK solution $(200 \mathrm{ml})$ at $0-4^{\circ} \mathrm{C}$ at a rate of $0.5 \mathrm{ml} / \mathrm{g} / \mathrm{min}$ as detailed previously (17).

Microscopy. Acridine orange (AO; Beijing Solarbio Science and Technology Co., Ltd., Beijing, China) binds to nucleic acids and stains viable cells green. However, propidium iodide (PI; Wuhan Goodbio Technology Co., Ltd., Wuhan, China), which has a higher affinity to nucleic acids, may enter cells and displace $\mathrm{AO}$ from the binding site, resulting in a clear red stain only, indicating when the cell is dead (18-20). To identify whether complete perfusion was achieved, HTK containing AO $(13.5 \mu \mathrm{M})$ and PI $(14.9 \mu \mathrm{M})$ was used as perfusate. During HMP, the cells in different areas of the rat liver were stained along with the perfusate distribution (viable cells were stained by AO, dead cells were stained by PI) as previously described (21). At the end of HMP, the liver was divided into core region (CR) and peripheral region (PR) (Fig. 2). Subsequently, samples of the two areas were immediately frozen in liquid nitrogen followed by serial cryosections (4 $\mu \mathrm{m}$ thick) for fluorescence microscopy. The cryosections were used to examine $\mathrm{AO}$ and PI positive cells in the samples at 495/519 nm (fluorescein isothiocyanate) and 547/572 nm (tetramethylrhodamine) filters using an inverted fluorescence microscope (Nikon Eclipse TI-SR; Nikon Corporation, Tokyo, Japan). Each specimen was counted in 3 random fields at a magnification of $\mathrm{x} 200$. AO and PI positive cells were counted 

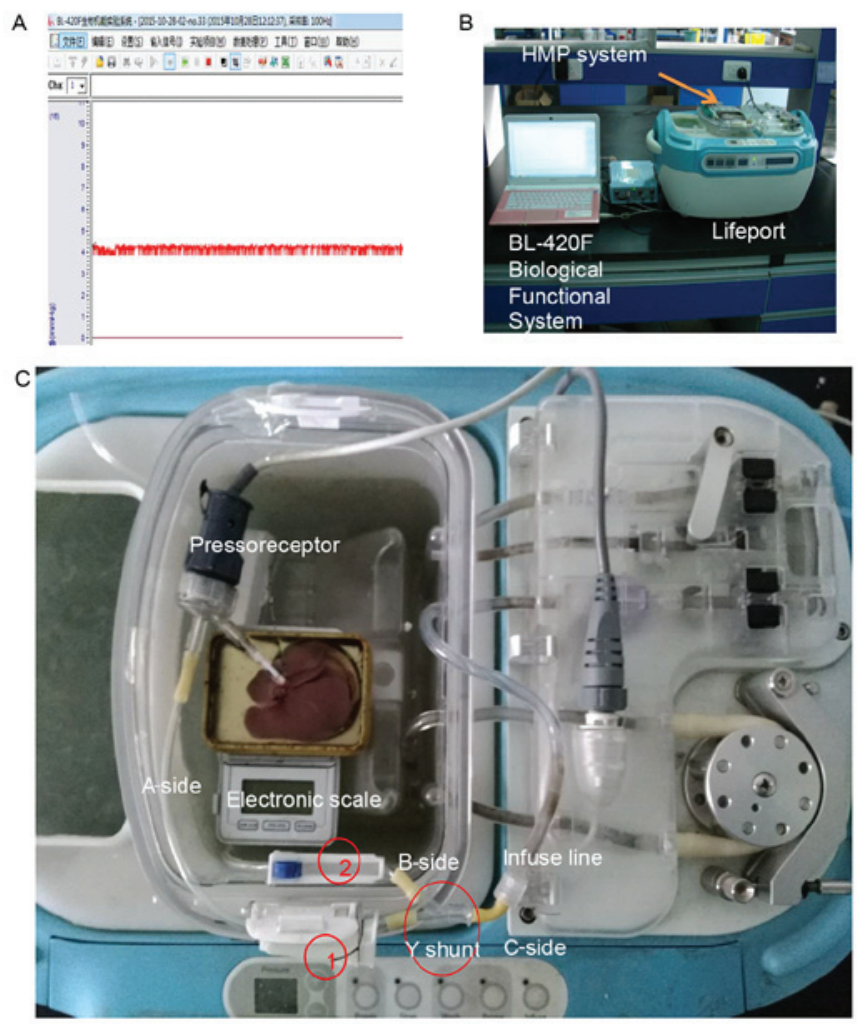

Figure 1. Modified HMP system using a LifePort Kidney Transporter for preserving rat livers. (A) Perfusion oscillogram with high frequency and small wave range under the prime mode of the LifePort. (B) Overall appearance of the modified HMP system. (C) The modified system installed on the LifePort. HMP, hypothermic machine perfusion.

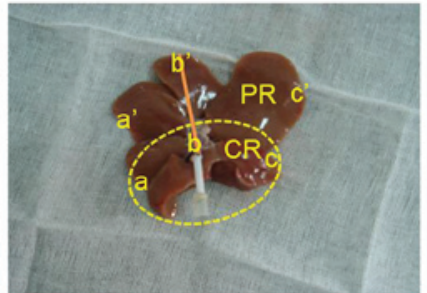

Anterior view

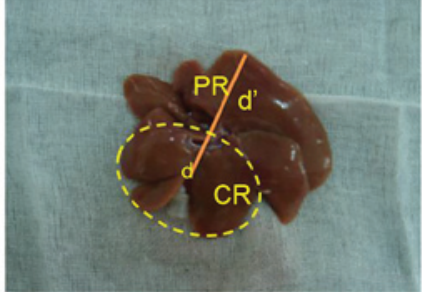

Posterior view
Figure 2. Division of liver and method for obtaining samples. A straight line was drawn from the entrance of portal vein to the furthest point of liver periphery and a circle, whose center was the entrance of portal vein and radius was $1 / 3$ of the straight line, was drawn to divide the liver into the CR and PR. At the end of hypothermic machine perfusion, samples at points a, b, c and d of CR and a', b', c' and d' points of PR were obtained. CR, core region; PR, peripheral region.

in each field. AO+PI-positive cell count was recorded and analyzed using Image-Pro Plus 6.0 (Media Cybernetics, Inc., Rockville, MD, USA).

To assess changes in morphology, samples were obtained and analyzed via hematoxylin and eosin staining of a peripheral biopsy following the completion of flush in situ (pre-HMP sample) and a peripheral biopsy at the end of HMP (post-HMP sample). The samples obtained were fixed in formalin solution $(10 \%)$ at room temperature for $24 \mathrm{~h}$. Next, the fixed samples were serially cut into sections $(5 \mu \mathrm{m})$, and stained by hematoxylin and eosin (hematoxylin staining for 5-15 min, eosin staining for 1-3 min, staining was performed at room temperature). Finally, the samples were observed under a light microscope. The levels of sinusoidal dilatation, endothelial cell detachment and vacuolization were determined by two independent operators. Sections were analyzed using light microscopy of $\geq 15$ random microscopic fields at a magnification of $\times 200$ from three separate biopsies of pre-HMP and post-HMP samples, and the images were captured using the Nikon Eclipse TI-SR microscope. The images were analyzed using the point-counting method within grids that contained 80 points combined with ImageJ 1.42 software (National Institutes of Health, Bethesda, MD, USA) (22). Every point was recorded for the incidence of sinusoidal dilatation, sinusoidal endothelial cell detachment, vacuolization, and the percentage in each observation was calculated, as described previously (22).

Data management and sample collection. The flow, pressure, intrahepatic resistance (IR), and temperature were recorded every $3 \mathrm{~h}$ for a total of $6 \mathrm{~h}$ during HMP. IR was calculated according to the following formula: $\mathrm{IR}(\mathrm{mmHg} / \mathrm{ml} / \mathrm{min})=$ portal pressure $(\mathrm{mmHg}) /$ portal flow $(\mathrm{ml} / \mathrm{min})$.

Hepatic effluent was collected from the catheter cannulated in the suprahepatic vena cava every $3 \mathrm{~h}$ of the $6-\mathrm{h}$ HMP period and the levels of alanine transaminase (ALT) and lactate dehydrogenase (LDH) were analyzed. Enzymatic activities were detected using alanine aminotransferase assay kit (cat no. C009-2) and lactate dehydrogenase assay kit (cat no. A020-2) (both from Nanjing Jiancheng Bioengineering Institute) according to the manufacturer's protocols.

Detection of endothelin was performed to determine the level of shear stress and pressure damage to the endothelial cell layer due to HMP. The level of endothelin in the hepatic effluent every $3 \mathrm{~h}$ of the 6-h HMP period was assessed using a commercialized assay kit (cat no. H093; Nanjing Jiancheng Bioengineering Institute) in accordance with the manufacturer's protocol.

Oxygen consumption (OC) is a marker of hepatic metabolic activity (10). Perfusate samples from the portal inflow and the catheter cannulated in suprahepatic vena cava were respectively collected and measured using a pH-blood gas analyzer (i-STAT; Abbott Point of Care, Inc., Princeton, NJ, USA) and the OC was calculated in accordance with the formulas detailed previously (23). The oxygen solubility in water at $4^{\circ} \mathrm{C}\left(\mathrm{SO}_{2}{ }^{4 \mathrm{C}}\right)=0.02112 \mathrm{mmol} \mathrm{O} \mathrm{O}_{2} / \mathrm{ml} \mathrm{x} \mathrm{kPa} \mathrm{(24).}$

Bile was collected from the tube placed in the common bile duct at the end of HMP. As the density of bile and water was equal, the bile flow was gravimetrically calculated and expressed as $\mu \mathrm{l} / \mathrm{h} / \mathrm{g}$ of liver following normalization of liver weight.

Assessment of ATP content in rat livers. Liver tissues stored at $-80^{\circ} \mathrm{C}$ were homogenized using $4.2 \%$ perchloric acid and $1 \mathrm{mM}$ diethylenetriamine pentaacetic acid prior to centrifugation at 14,000 x g (4 C, 5-10 min). Subsequently, the supernatants were obtained and mixed with $69 \% \mathrm{~K}_{2} \mathrm{CO}_{3}$ ( $\mathrm{pH}$ 6). The content of ATP in liver samples was measured using an ATP commercial assay kit (cat no. A095-1; Nanjing Jiancheng Bioengineering Institute) according to the manufacturer's instructions. 
Statistical analysis. Data were presented as the mean \pm standard error of the mean. The differences between the dynamic perfusion parameters at three analyzed time points $(0,3$ and $6 \mathrm{~h}$ ) were analyzed using one-way analysis of variance and the Least Significant Difference test for multiple comparisons. The comparison of $\mathrm{AO}+\mathrm{PI}-$ positive cell count between $\mathrm{CRs}$ and PRs were analyzed by Student's t-test. Histological scores of pre-HMP and post-HMP tissues were tested by Student's t-test. $\mathrm{P}<0.05$ was considered to indicate a significant difference.

\section{Results}

Perfusion status and dynamics. AO+PI-positive cell count between the CR and PR at the end of HMP was used to identify the perfusion status. As Fig. 3 indicates, no significant difference was observed between the CR and PR when the AO+PI-positive cell count was analyzed, which indicates that completed perfusion was achieved at a perfusion flow of $0.5 \mathrm{ml} / \mathrm{g} / \mathrm{min}$.

During HMP, although a significant decrease was observed between $0(0.95 \pm 0.08 \mathrm{mmHg} / \mathrm{ml} / \mathrm{min})$ and $3 \mathrm{~h}$ $(0.24 \pm 0.04 \mathrm{mmHg} / \mathrm{ml} / \mathrm{min})$ of perfusion $(\mathrm{P}<0.01$; Fig. $4 \mathrm{~A})$, IR levels remained constant between $3(0.24 \pm 0.04 \mathrm{mmHg} / \mathrm{ml} / \mathrm{min})$ and $6(0.17 \pm 0.05 \mathrm{mmHg} / \mathrm{ml} / \mathrm{min})$ h of perfusion.

Assessment of hepatocyte viability, metabolic activity and cellular injury associated with HMP shear stress injury. The release of ALT and LDH enzymes was used to assess hepatocyte viability during HMP. A significant increase of the levels of ALT and LDH enzymes were observed during HMP $(\mathrm{P}<0.01$; Fig. 4B and $\mathrm{C}$, respectively). The levels of ALT at 0 , 3 and $6 \mathrm{~h}$ were $42.67 \pm 5.81,90.67 \pm 6.74$ and $164.33 \pm 7.31 \mathrm{U} / 1$, respectively (Fig. 4B), and the levels of LDH at 0,3 and $6 \mathrm{~h}$ were $492.90 \pm 90.20,973.53 \pm 97.42$ and $1,843.40 \pm 85.78 \mathrm{U} / 1$, respectively (Fig. 4C).

Endothelin secreted from sinusoidal endothelial cells is capable of reflecting cellular shear stress and pressure response induced by HMP (25). Results indicated that the levels of endothelin at 0,3 and $6 \mathrm{~h}$ were $0.038 \pm 0.010,0.031 \pm 0.005$ and $0.046 \pm 0.004 \mathrm{fmol} / \mathrm{ml}$, respectively (Fig. 4D), which were not significantly different.

OC, ATP production and bile production were markers to assess cellular metabolic activity. It has been reported that $\mathrm{OC}$ of the normal rat liver during warm reperfusion was $1.95 \pm 0.78 \mu \mathrm{mol} \mathrm{O} / \mathrm{min}^{-1} \cdot \mathrm{g}^{-1}$ liver (23). Compared with these results, the levels of $\mathrm{OC}$ in our experiment were lower but constant over the 6-h time period of HMP (Fig. 4E; $0 \mathrm{~h}$, $0.76 \pm 0.16 ; 3 \mathrm{~h}, 0.66 \pm 0.09 ; 6 \mathrm{~h}, 0.71 \pm 0.19 \mu \mathrm{mol} \mathrm{O}_{2} / \mathrm{min}^{-1} . \mathrm{g}^{-1}$ liver; P>0.05). Furthermore, the levels of ATP and bile production at the end of HMP were $640.64 \pm 29.46 \mathrm{nmol} / 1$ and $4.88 \pm 0.69 \mu \mathrm{l} / \mathrm{h} / \mathrm{g}$ of liver, respectively.

Liver histology. Liver samples were analyzed using hematoxylin and eosin staining (Fig. 5A and B). Sinusoidal dilatation results indicated widening of sinusoids was significantly increased following HMP when compared with pre-HMP $(\mathrm{P}<0.01$; Fig. 5C). However, endothelial cell detachment, which is a major cellular damage associated with HMP (10,21), did not significantly differ between the pre- and post-HMP groups (Fig. 5C). Furthermore, vacuolization has

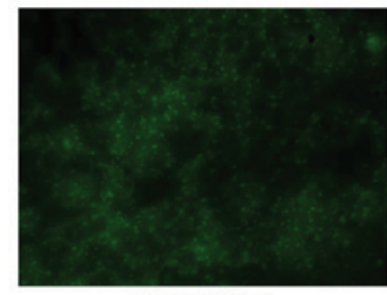

AO staining

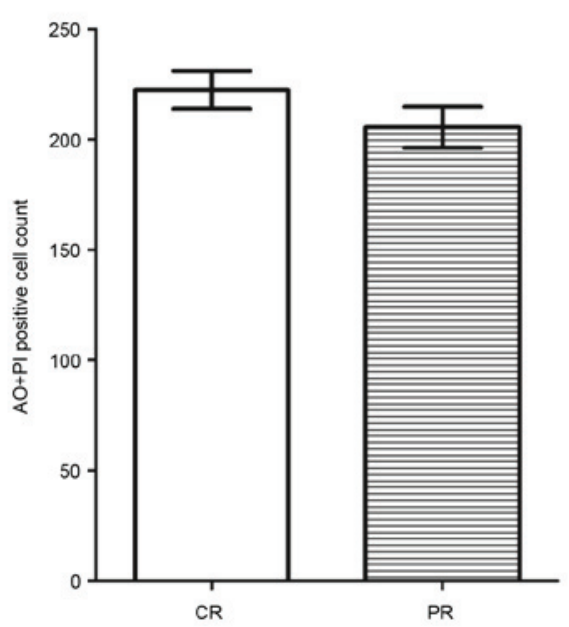

Figure 3. AO and PI staining in the liver. AO was used to stain viable cells a green, whereas dead cells were detected by red PI staining. AO+PI-positive cell count was calculated in the $\mathrm{CR}$ and $\mathrm{PR}$ of the rat liver. No significant difference was observed between the two regions. Data are presented as the mean \pm standard error of mean. AO, acridine orange; PI, propidium iodide; $\mathrm{CR}$, core region; $\mathrm{PR}$, peripheral region.

been demonstrated as a sign of cell injury associated with HMP $(10,21)$. However, no elevated incidence of vacuolization in the hepatocytes of post-HMP samples was observed compared with pre-HMP (Fig. 5C).

\section{Discussion}

The present study aimed to demonstrate the feasibility of a HMP system with a LifePort Kidney Transporter for preserving rat livers. Results suggest that the modified HMP system may meet the demands of the flow and pressure under the hypothermic conditions that are suitable for rat liver preservation, while retaining the dependability and portability of the LifePort Kidney Transporter.

Rat livers were continuously perfused with HTK solution via the portal vein at $0-4^{\circ} \mathrm{C}$ at a rate of $0.5 \mathrm{ml} / \mathrm{g} / \mathrm{min}$, which has been previously demonstrated to allow complete and homogeneous perfusion with minimal cellular injury in rat livers following HMP (25). In the present study, HTK containing $\mathrm{AO}$ and PI was used as perfusate to determine the perfusion status via staining cells. Results indicated no significant differences between the CR and PR in the liver samples according to the comparison of the AO+PI-positive cell count at the end of HMP, which indicated that the complete and homogeneous distribution of perfusate was achieved using the present modified HMP system with a LifePort Kidney Transporter.

Cold ischemia and physical injury due to HMP are major cellular damages that occur during the perfusion period (21). 


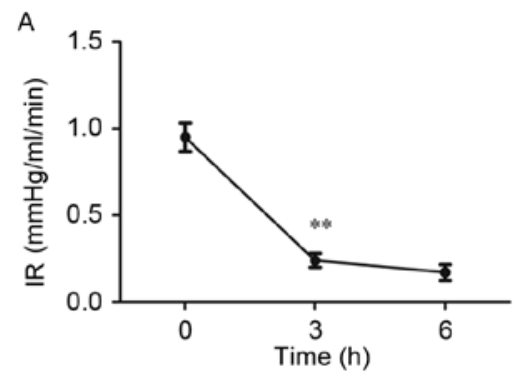

D

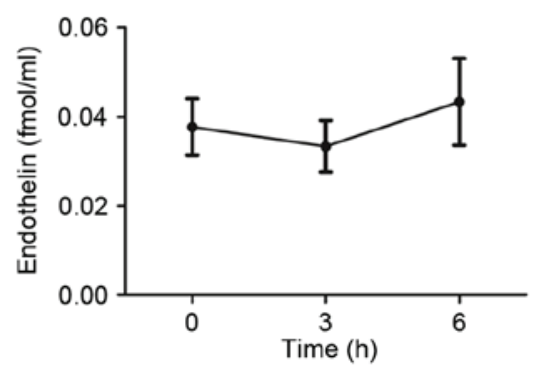

B

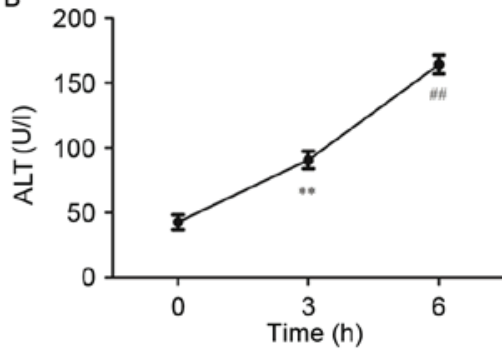

$\mathrm{C}$

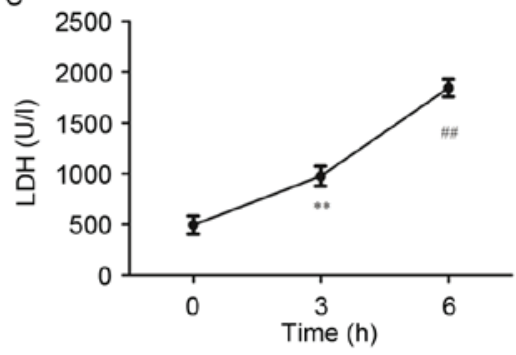

E

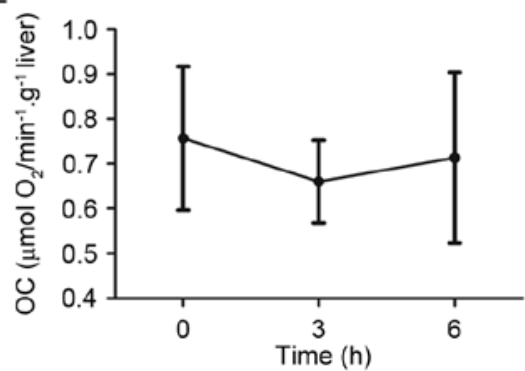

Figure 4. Assessment for the liver during HMP at 0,3 and $6 \mathrm{~h}$. (A) A significant decrease of IR level was observed in the first $3 \mathrm{~h}$ of HMP; however, the IR level was maintained between 3 and $6 \mathrm{~h}$ of HMP. (B) ALT and (C) LDH levels were significantly increased during HMP. No significant difference was observed in (D) endothelin release and (E) OC over the 6-h HMP period. Data are presented as the mean \pm standard error of the mean $(10 \mathrm{samples}) .^{* *} \mathrm{P}<0.01 \mathrm{vs} .0 \mathrm{~h}$, ${ }^{\# \#} \mathrm{P}<0.01$ vs. 3 h. HMP, hypothermic machine perfusion; IR, intrahepatic resistance; OC, oxygen consumption; ALT, alanine transaminase; LDH, lactate dehydrogenase.

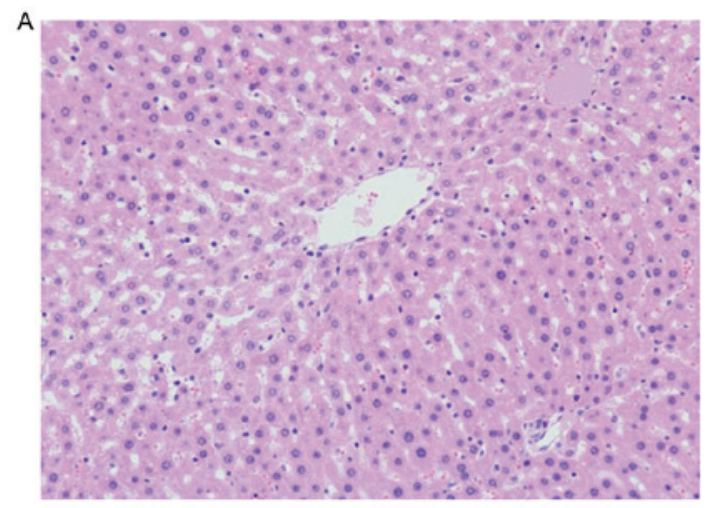

C

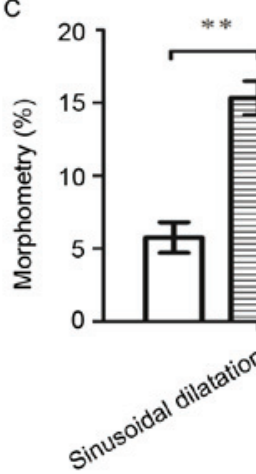

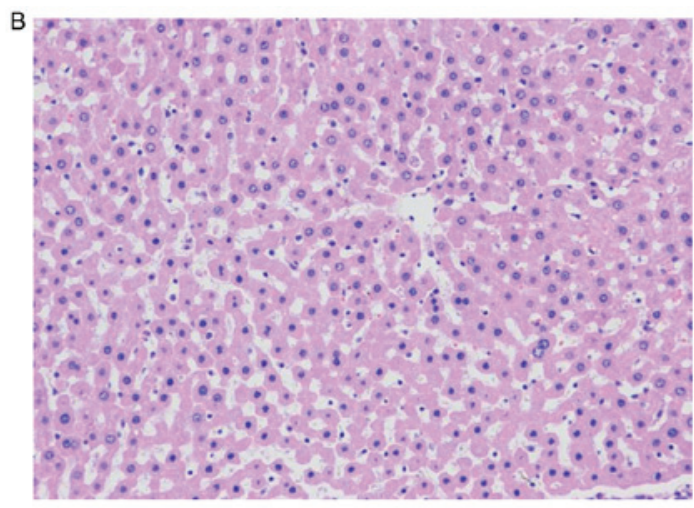

$\square$ Pre-HMP

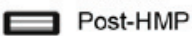

Figure 5. Representative histological findings and morphometric analysis of pre- and post-HMP samples. Representative images of (A) pre-HMP and (B) post-HMP samples stained with hematoxylin and eosin findings were viewed using a light microscope (magnification, $\mathrm{x} 200$ ). (C) Morphometric analysis suggested that post-HMP samples had a significantly increased percentage of sinusoidal dilatation compared with pre-HMP samples; although no significant aggravation of endothelial cell detachment and hepatocellular vacuolization was observed. Data are presented as the mean \pm standard error of the mean (10 samples). ${ }^{* *} \mathrm{P}<0.01$. HMP, hypothermic machine perfusion.

Therefore, to assess the extent of cellular damage, biochemical and histological parameters were investigated to judge the damage profile due to HMP. During HMP, although the levels of ALT and LDH were significantly increased over the total 
6-h time period, endothelin release, which is typically used to reflect the levels of cellular shear stress and pressure caused by HMP (25), did not significantly increase. These findings were in accordance with those observed previously in similar recirculating perfusion devices $(12,25)$ and indicated the shear stress and pressure injury of the present HMP system was minimal, and that the increased ALT and LDH release was likely due to cold ischemia injury. Similarly, histological observations indicated the general morphology of the parenchyma was preserved and sinusoidal dilatation was significantly increased in post-HMP tissues compared with pre-HMP tissues. In addition, the maintained hepatic OC levels, and ATP and bile production observed in the present study, which were consistent with a previous study using a similar HMP system (12), suggested that the cellular metabolic activity was effectively preserved following HMP preservation for $6 \mathrm{~h}$. Therefore, it was concluded that HMP of rat livers resulted in complete and homogeneous perfusion at a rate of $0.5 \mathrm{ml} / \mathrm{g} / \mathrm{min}$ with HTK solution using the modified HMP system, and cellular injury resulting from shear stress and pressure of HMP was minimal. These findings were in accordance with other previous studies $(12,21,25)$ and indicated that the modified HMP system with the LifePort Kidney Transporter worked effectively.

The primary concern in the use of the LifePort for HMP preservation of rat liver is that the size of the infusion line is not suitable for the rat portal vein. To overcome this obstacle, a self-made ' $Y$ ' shunt was designed to connect the portal vein and the infuse line of the LifePort based on their pipe sizes. The large side of the ' $\mathrm{Y}$ ' shunt was connected with infuse line and the small side matched with the rat portal vein.

Continuous HMP via the portal vein for liver preservation is capable of simulating hemodynamics of the portal vein and has been widely applied in a number of previous studies $(12,21,25)$. However, continuous perfusion under hypothermic conditions using the perfusion mode of LifePort was not able to be performed. By contrast, according to the preliminary results in the present study, the prime mode of Lifeport could achieve continuous perfusion, which was indicated by the oscillogram with a high frequency and small wave range.

Prevention of excessive perfusion pressure is necessary to protect organs in a LifePort transporter. HMP may immediately terminate once the perfusion pressure exceeds the upper limit of the LifePort. The increase of perfusion resistance due to the relatively small volume of rat liver and large flow of the LifePort using prime mode typically initiates the prevention function and causes the machine to stop. Therefore, a ' $\mathrm{Y}$ ' shunt was constructed to decrease the portal inflow and perfusion pressure in the present study.

The final problem needed to be solved was the measurement of portal inflow. An accurate portal inflow measurement cannot be made due to the lack of a display for flow under prime mode in LifePort and the use of the ' $\mathrm{Y}$ ' shunt. Therefore, an electronic scale under the liver container was installed in the present study and the change of weight per min during HMP was measured. Subsequently, the portal inflow per min was calculated according to the association with the volume, weight and density of perfusate.

In general, Lifeport stops automatically once excessive pressure occurs. However, the limitation of our modification was that this prevention function of Lifeport could no longer be properly initiated. Therefore, the operator was required to monitor the change of pressure prior to IR stabilization, particularly at the beginning of HMP.

In previous studies, the HMP system for rat livers typically contained a roller pump, liver container, a bubbler to deliver air, a flowmeter to measure flow, a nylon filter to prevent the recirculation of cellular debris and blood clots, a water column to measure the perfusion pressure, and a cooling system to maintain the hypothermic condition for perfusion $(12,21,25)$. The lack of effective integration of these equipment was the major obstacle associated with the portability of HMP, leading to restriction for its implementation. In the present study, a simple modification was made to the LifePort Kidney Transporter to ensure HMP for rat liver while retaining portability.

In conclusion, the present study demonstrated the feasibility of a modified HMP system using a LifePort Kidney Transporter for rat liver preservation. Although the automation of this system requires further modification, the present results suggest that, using the modified system, the demands of flow and pressure during continuous HMP via the rat portal vein can be met, while the dependability and portability of the LifePort are retained.

\section{Acknowledgements}

The present study was supported by National Natural Science Foundation of China (grant no. U1403222).

\section{References}

1. Henry SD, Nachber E, Tulipan J, Stone J, Bae C, Reznik L, Kato T, Samstein B, Emond JC and Guarrera JV: Hypothermic machine preservation reduces molecular markers of ischemia/reperfusion injury in human liver transplantation. Am J Transplant 12: 2477-2486, 2012

2. Mirza DF, Gunson BK, Da Silva RF, Mayer AD, Buckels JA and McMaster P: Policies in Europe on 'marginal quality' donor livers. Lancet 344: 1480-1483, 1994.

3. Serrano MT, Garcia-Gil A, Arenas J, Ber Y, Cortes L, Valiente C and Araiz JJ: Outcome of liver transplantation using donors older than 60 years of age. Clin Transplant 24: 543-549, 2010.

4. Frühauf NR, Fischer-Fröhlich CL, Kutschmann $M$, Schmidtmann I and Kirste G: Joint impact of donor and recipient parameters on the outcome of liver transplantation in Germany. Transplantation 92: 1378-1384, 2011.

5. Blok JJ, Detry O, Putter H, Rogiers X, Porte RJ, van Hoek B, Pirenne J, Metselaar HJ, Lerut JP, Ysebaert DK, et al: Longterm results of liver transplantation from donation after circulatory death. Liver Transpl 22: 1107-1114, 2016.

6. Nemes B, Gaman G, Polak WG, Gelley F, Hara T, Ono S, Baimakhanov Z, Piros L and Eguchi S: Extended criteria donors in liver transplantation Part I: Reviewing the impact of determining factors. Expert Rev Gastroenterol Hepatol 10: 827-839, 2016.

7. Casavilla A, Ramirez C, Shapiro R, Nghiem D, Miracle K, Bronsther O, Randhawa P, Broznick B, Fung JJ and Starzl T: Experience with liver and kidney allografts from non-heart-beating donors. Transplantation 59: 197-203, 1995.

8. Minor T, Akbar S, Tolba R and Dombrowski F: Cold preservation of fatty liver grafts: Prevention of functional and ultrastructural impairments by venous oxygen persufflation. J Hepatol 32: 105-111, 2000.

9. Bae C, Henry SD and Guarrera JV: Is extracorporeal hypothermic machine perfusion of the liver better than the 'good old icebox'? Curr Opin Organ Transplant 17: 137-142, 2012.

10. Carnevale ME, Balaban CL, Guibert EE, Bottai $\mathrm{H}$ and Rodriguez JV: Hypothermic machine perfusion versus cold storage in the rescuing of livers from non-heart-beating donor rats. Artif Organs 37: 985-991, 2013. 
11. Guarrera JV, Henry SD, Samstein B, Odeh-Ramadan R, Kinkhabwala M, Goldstein MJ, Ratner LE, Renz JF, Lee HT, Brown RS Jr and Emond JC: Hypothermic machine preservation in human liver transplantation: The first clinical series. Am J Transplant 10: 372-381, 2010.

12. Jia JJ, Zhang J, Li JH, Chen XD, Jiang L, Zhou YF, He N, Xie HY, Zhou L and Zheng SS: Influence of perfusate on liver viability during hypothermic machine perfusion. World J Gastroenterol 21: 8848-8857, 2015.

13. Gores GJ, Kost LJ and LaRusso NF: The isolated perfused rat liver: Conceptual and practical considerations. Hepatology 6: 511-517, 1986.

14. Moser M, Sharpe M, Weernink C, Brown H, McGregor T, House AA and Luke PP: Five-year experience with donation after cardiac death kidney transplantation in a Canadian transplant program: Factors affecting outcomes. Can Urol Assoc J 6: 448-452, 2012.

15. Wszola M, Kwiatkowski A, Diuwe P, Domagała P, Górski L, Kieszek R, Berman A, Perkowska-Ptasińska A, Durlik M, Pączek L and Chmura A: One-year results of a prospective, randomized trial comparing two machine perfusion devices used for kidney preservation. Transpl Int 26: 1088-1096, 2013.

16. Kamada N and Calne RY: A surgical experience with five hundred thirty liver transplants in the rat. Surgery 93: 64-69, 1983.

17. Minor T, Manekeller S, Sioutis M and Dombrowski F: Endoplasmic and vascular surface activation during organ preservation: Refining upon the benefits of machine perfusion. Am J Transplant 6: 1355-1366, 2006.
18. Foglieni C, Meoni C and Davalli AM: Fluorescent dyes for cell viability: An application on prefixed conditions. Histochem Cell Biol 115: 223-229, 2001.

19. Bank HL: Assessment of islet cell viability using fluorescent dyes. Diabetologia 30: 812-816, 1987.

20. Bank HL: Rapid assessment of islet viability with acridine orange and propidium iodide. In Vitro Cell Dev Biol 24: 266-273, 1988.

21. t Hart NA, der van Plaats A, Leuvenink HG, van Goor $\mathrm{H}$, Wiersema-Buist J, Verkerke GJ, Rakhorst G and Ploeg RJ: Determination of an adequate perfusion pressure for continuous dual vessel hypothermic machine perfusion of the rat liver. Transpl Int 20: 343-352, 2007.

22. Pizarro MD, Rodriguez JV, Mamprin ME, Fuller BJ, Mann BE, Motterlini R and Guibert EE: Protective effects of a carbon monoxide-releasing molecule (CORM-3) during hepatic cold preservation. Cryobiology 58: 248-255, 2009.

23. Balaban CL, Rodriguez JV and Guibert EE: Delivery of the bioactive gas hydrogen sulfide during cold preservation of rat liver: Effects on hepatic function in an ex vivo model. Artif Organs 35: 508-515, 2011.

24. Graham M: The solubility of oxygen in physiological salines. Fish Physiol Biochem 4: 1-4, 1987.

25. Lüer B, Koetting M, Efferz P and Minor T: Role of oxygen during hypothermic machine perfusion preservation of the liver. Transpl Int 23: 944-950, 2010 .

(i) (3) This work is licensed under a Creative Common

Attribution-NonCommercial-NoDerivatives 4.0 International (CC BY-NC-ND 4.0) License. 